\title{
Can More than One Fistula Be Treated by a New Type of Seton Concomitantly?
}

\author{
Bülent Erenoğlu, Kemal Arslan*, Hande Köksal, Osman Doğru \\ Department of General Surgery, Konya Training and Research Hospital, Konya, Turkey \\ Email: "arslanka74@hotmail.com
}

Received November 2, 2012; revised December 4, 2012; accepted December 13, 2012

\begin{abstract}
Objective: The treatment of trans-sphincteric fistulas is a surgical challenge since eradicating the fistula tract while maintaining the anal continence is vitally important. In this study we documented the outcomes of the patients with one or more fistulas treated at the same operation with a new type of seton. Patients and Methods: The study included 78 patients with 107 high anal fistulas who were treated with seton between 2007 and 2011. Fifty seven patients had only one fistula, 13 patients had two isolated fistulas and 8 patients had three isolated fistulas. Group I was the patients with one fistula and Group II was the patients having more than one fistula. Postoperative complications, hospital stay, cutting through the sphincter and healing times and Wexner's scores were compared between the two groups. Results: In Group I, there were 57 patients and in Group II, 21 patients were operated for 50 fistulas. No postoperative complications were observed except urinary retentions (4 patients in Group I, 3 patients in Group II). No infection on the operation site was documented in both groups, in Group I none of the patients needed extra dose of narcotic analgesic but in Group II two of the patients who had 3 fistulas were discharged on the second postoperative day because of pain on the operation site. The median for the cutting seton to cut through the sphincter was 31 days and complete healing was achieved with a median of 40 days in Group I, whereas it was 56 and 65 days respectively for Group II. There were no recurrences in our patients in both groups. Although the Wexner scores of the both groups were low, the difference between the groups was statistically significant. Conclusion: It seems to be feasible to perform seton by using a cheap, effective, easily inserted material, also in treatment of patients with more than one fistula while preserving anal continence.
\end{abstract}

Keywords: Trans-Sphincteric Fistula; Seton; Multiple Perianal Fistül

\section{Introduction}

Most common cause of anal fistula is an anorectal abscess particularly if drained spontaneously or had been inadequately drained during the operation. Nearly all of the anal fistulas can be classified as simple fistula and can be safely treated by fistulotomy [1]. However treatment of high and complicated fistulas still remain as a challenge because of the probability of leading to anal incontinence and recurrence. For this reason it is important to choose correct surgical strategies to preserve the sphincter mechanism. Different kinds of setons have been used for this purpose for many years. In this study we documented the outcomes of the patients with one or more isolated fistulas treated at the same operation with a new type of seton recently introduced by Vatansev et al. [1].

\section{Patients and Methods}

Demographic features, operative findings and surgical

${ }^{*}$ Corresponding author. outcomes of the patients treated by cutting seton for high anal fistulas between 2007 and 2011 were analyzed retrospectively. Patients with inflammatory bowel diseases, malignities, endocrine disorders, previous anorectal surgeries and history of vaginal deliveries with tears or episiotomies were excluded. Anal physical examination findings and endoanal ultrasound records were used to determine the fistula tracts. The study included 78 patients with 107 high anal fistulas who were treated with seton. Of the 78 patients, 64 were men and 14 were women. Fifty seven patients had only one fistula, 13 patients had two fistulas and 8 patients had three fistulas. All of the patients with more than one fistula had isolated tracts and orifices apart from complex fistulas with more than one external orifice. The patients were classified into two groups according to the number of fistulas they had: Group I was the patients with one fistula and Group II was the patients having more than one fistula (Figure 1). All of the operations were under spinal anesthesia with the patient in the lithotomy position. In cases with undefined internal orificis, methylene blue or hydrogen 


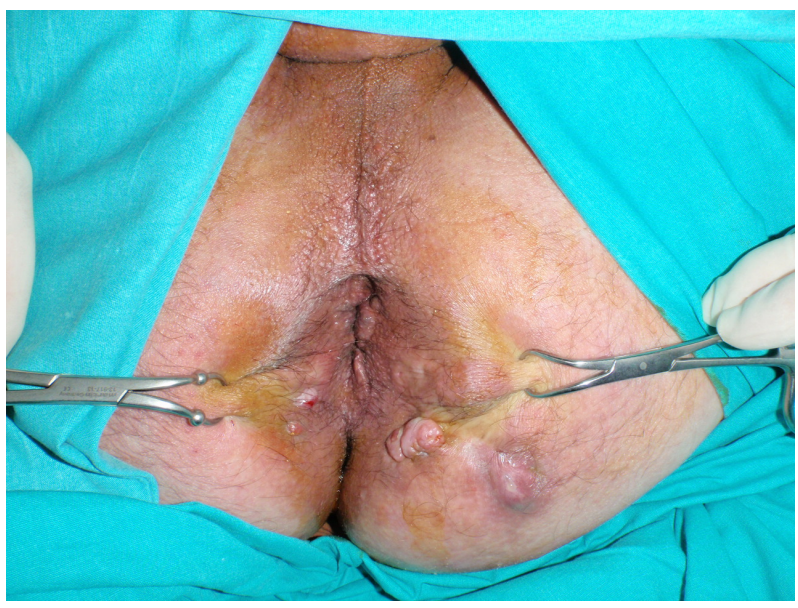

Figure 1. Patient with more than one anal fistulas, in lithotomy position.

peroxide was used. According to the fistulous tract's relation with the sphincter muscles, the kind of the operation was decided by the surgeon so cutting seton were used in all patients and all of the patients were operated by the same surgeon. Before the operation the patients used enema and single dose of metronidazol was used during the operation as prophylaxis. After removing the granulation tissue, the part of the tract outside the sphincter muscles were laid open and seton was inserted into the tract which had a close relationship with sphincter mechanism. The seton was then locked on the sphincter with a sweet-tight manner (Figures 2 and 3). The seton used for treatment is an industrial synthetic cable tie, which was recently introduced in medicine, $10 \mathrm{~cm}$ in length, $0.1 \mathrm{~cm}$ in width and a locking mechanism avoiding to loose on the head part. It was cheap, harmless and can be easily found. Once the seton was tightened it remained in place for at least 4 weeks with a control of once a week. In this period warm sitz baths after each bowel movement were advised and the patients were informed about the possible serous discharge. Postoperative anal incontinence scores (at sixth month) were assessed according to the Wexner's scoring system. Recurrence was defined as reappearance of the fistula at or close to the original tract after complete healing occurs. Statistical analysis was performed with SPSS for Windows, release 16.0. Results are expressed as median. Statistical analysis was performed using the Mann-Whitney $\mathrm{U}$ test. Statistical significance was assumed at a level of $p$ $<0.05$.

\section{Results}

In Group I, there were 57 patients, 45 were male and 12 were female (M/F: 45/12). The median age of the patients in Group I was 42 years ranging from 23 to $65 \mathrm{y}$. The location of the fistula was anterior trans-sphincteric

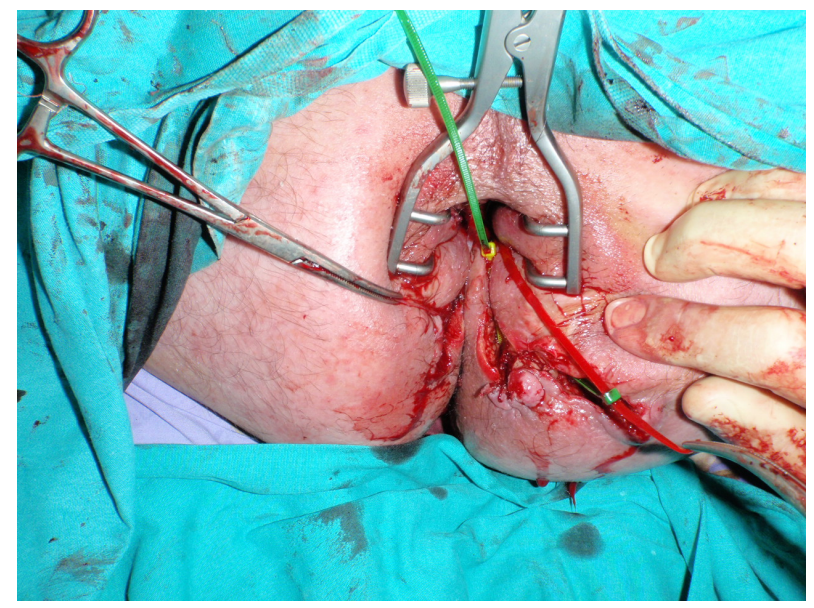

Figure 2. Patient with multiple setons inserted at the operation.

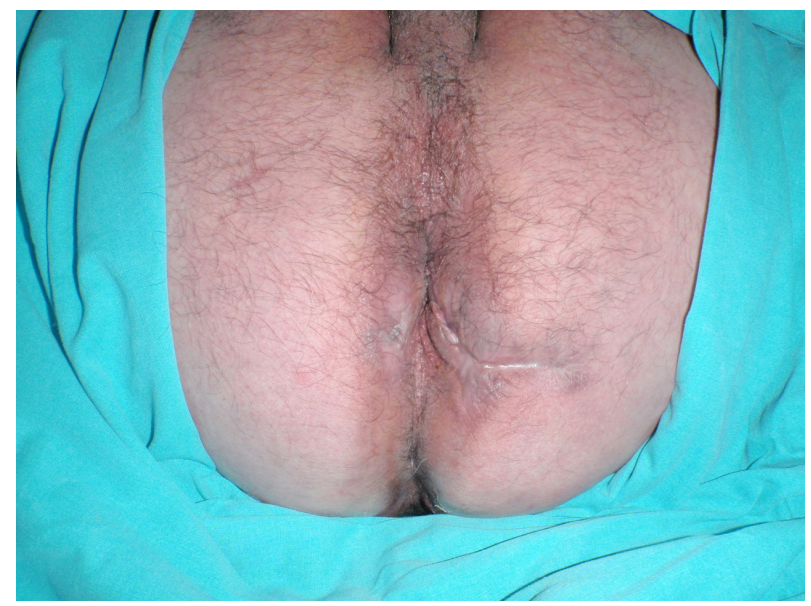

Figure 3. Patient after healing occurs.

in 52 patients and lateral high trans-sphincteric in 5 patients. No postoperative complications were observed except $4(7 \%)$ urinary retentions. All of the patients were discharged on the first postoperative day. None of the patients needed narcotic analgesics after discharge. No infection on the operation site was documented. The median for the cutting seton to cut through the sphincter was 31 days ranging between 28 and 36 days. Complete healing was achieved between 35 and 46 days with a median of 40 days. The follow-up time was between 24 and 40 months with a median of 30 months. No recurrence was seen in any patient. Postoperative incontinence score was between 1 and 2 with a median of 1 (mean value: $1.19 \pm 1$ ).

In Group II, 21 patients were operated for 50 fistulas, all of them by the same surgeon. Thirteen of them had 2 fistulas and 8 of them had 3 fistulas. Of the 21 patients 19 were male and 2 were female. The median age in Group II was 44 years (between 26 and 58 y). In three (14\%) patients, urinary retentions were recorded. Two of 
the patients who had 3 fistulas were discharged on the second postoperative day because of pain on the operation site and they needed additional single dose of narcotic analgesic. No infection was documented. The median cutting time for the seton to cut through the sphincter was 56 days ranging between 45 and 60 days. In this group complete healing was achieved in longer time with a median of 65 days (range: 58 - 75 days). The statistical difference between Group I and Group II was significant $(p=0.0001)$. When the patients in Group II were divided into two subgroups according to the number of fistulas they had, the subgroup with 2 fistulas had a median cutting time of 56 days (range: 45 - 58 days) and complete healing time of 65 days as a median (range: 58 - 67 days), while 3 fistulas subgroup had a median cutting time of 58.5 days (range: 50 - 60 days) and median complete healing time of 68.5 days (range: 65 - 75 days). The difference between the two subgroups was also statistically significant $(p=0.004$ for cutting time, $p=0.017$ for complete healing time). The follow-up time was between 18 and 31 months with a median of 26 months. No recurrence was mentioned in the follow-up period. Postoperative incontinence score was ranging between 1 and 5 (median: 2, mean: $2.43 \pm 1.12$ ). Although the Wexner scores of the both groups were low, the difference between the groups was statistically significant $(\mathrm{p}=$ $0.0001)$.

\section{Discussion}

Anal fistulas have an incidence of 12.3 per 100,000 in men and 5.6 per 100,000 in women (3). Majority of our patients were men also. Intersphincteric gland infection is accepted to be the initiating event. The most common cause of anal fistula is anorectal abscess particularly drained spontaneously or had been inadequetly drained during the operation as in our patients. Other causes are trauma, fissures, previous rectal or gynaecological operations, tuberculosis, ulcerative colitis, Crohn's disease, anal canal carcinoma and rarely parasitosis like Enterobius vermicularis [1]. Anal fistulas can be classified mostly as simple by Parks classification, which is based on the relationship of the fistula with the external anal sphincter, and can be treated easily by fistulotomy [3]. Especially high trans-sphincteric fistulas, those above the dentate line, remain a surgical challenge because incontinence may result while dividing the external sphincter. For this reason it is important to choose the correct surgical strategy to preserve the sphincter mechanism. The first step in the successful treatment is careful assessment. Physical examination should be repeated under anaesthesia to evaluate the relationship of the fistul tract with the sphincter muscles. If the surgeon can not define the extend of the tract, injection of hydrogen peroxide from the external openning will form bubble in the anal canal just at the level of internal openning. Fistulography, endoanal ultrasound, computed tomography or pelvic magnetic resonance imaging can also be used at the time of failure to recognize by physical examination. In management of anal fistulas the aim is to eradicate the septic focus and the tract while avoiding disturbing anal continence. Surgical procedures for treating high trans-sphincteric fistulas include mucosal advancement flap, fistulectomy, cutting setons, fistula excision, sphincteroplasty, injection of fibrin glue and plugs. The success rates of all these procedures are variable; recurrence after advancement flap closure is reported to be between $0 \%$ and $63 \%$, between $22 \%$ and $39 \%$ in cutting settons, and ranges from $14 \%$ to $60 \%$ in fibrin glue [4-6]). The seton works by several mechanisms; once it assists in identifying the fistula tract, it helps in draining pus and controlling the sepsis before the definitive treatment, by causing chronic inflammatory reaction and consequently stimulating fibrosis it prevents division and retraction of sphincter muscles which avoids anal incontinence [7]. Type of the seton selected depends on the personal preference as all of them have their own advantages and disadvantages. Chemical setons, drainage setons, cutting setons and two staged seton fistulotomy techniques can all be used for treating the complicated anal fistulas [8]. The aim of our study is to evaluate the results of the patients with one or more anal fistulas treated at the same operation with a different type of seton. In this study we used a different kind of seton for concomitant fistulas which was cheap, easy to find, simple to use, non-toxic and non-allergic, comfortable for the patient and having a self locking system [2]. In our study no postoperative complications were observed except urinary retentions (4 patients in Group I, 3 patients in Group II) which might be due to the spinal anesthesia. The higher urinary retention rate in Group II might be connected with the longer operation and anaesthetized time. No infection on the operation site was documented in both groups, which can be explained by using enema before the surgery and a single dose of metronidazol during the operation as prophylaxis. After the operation all of the patients were taught to have warm sitz baths for its cleaning and analgesic effect for the surgery site. In Group I none of the patients needed extra dose of narcotic analgesic but in Group II two of the patients who had 3 fistulas were discharged on the second postoperative day because of pain on the operation site and they needed additional single dose of narcotic analgesic. We think that this need for additional dose of analgesic was related with the number of the fistulas with tied-up setons. Since anorectal region have a great innervation, as the number of setons increases, the pain will increase undoubtedly.

The median for the cutting seton to cut through the sphincter was 31 days and complete healing was ach- 
ieved with a median of 40 days in Group I, whereas it was 56 and 65 days respectively for Group II. The differences between the two groups were significant and we think it was also related with the number of fistulas because each of them was a unique healing site. In patients with more than one fistula we had to behave slowly about tying up the seton in order to avoid cutting the sphincters quickly which leads to incontinence. So the cutting times of the seton in Group II were longer than those in Group I. This difference can also be seen between the two subgroups of Group II which supports our hypothesis about the relation between the cutting time, healing time and number of fistulas.

In the literature the recurrence rate of treatment with cutting seton is reported between $2 \%$ and $8 \%$ [9]. There were no recurrences in our patients in both groups which might be a wonderful result but it might be due to the short follow-up times also.

The reported rate of total incontinence disorders is about $60 \%$; flatus was reported to be $36.2 \%$, liquid stool was $8.5 \%$ and solid stool incontinence was reported to be $2.3 \%[10,11]$. In our study although the Wexner scores of the both groups were low, the difference between the groups was statistically significant $(p=0.0001)$. This statistical difference can be ignored because after this kind of anorectal operations Wexner score up to 5 can be accepted as mildly high [12]. None of the patients had severe incontinence in both groups and also baseline continence was not significantly disturbed. The fact that we had good results about recurrence, incontinence and other complications may be due to none of our patients had inflammatory bowel disease.

In patients with trans-sphincteric fistulas using seton is known to be a good surgical choice with favorable results and in this study we think that it is feasible to perform this surgical method by using a cheap, effective, easily inserted material also in treatment of patients with more than one fistula while preserving anal continence.

\section{REFERENCES}

[1] K. M. B. Dunn and D. A. Rothenberger, "Colon, Rectum and Anus. Schwartz's Principles of Surgery," 9th Edition, McGraw-Hill Companies, Inc., New York, 2010.

[2] C. Vatansev, O. Alabaz, A. Tekin, F. Aksoy, H. Y1lmaz, T. Kucukkartallar, T. Akcam and A. Pamukcu, "A New
Seton Type for the Treatment of Anal Fistula," Digestive Diseases and Sciences, Vol. 52, No. 8, 2007, pp. 1920 1923. doi:10.1007/s10620-007-9793-5

[3] A. Michalopoulos, V. Papadopoulos, N. Tziris and S. Apostolidis, "Perianal Fistulas," Techniques in Coloproctology, Vol. 1, Suppl. 1, 2010, pp. S15-S17. doi:10.1007/s10151-010-0607-y

[4] G. N. Buchanan, H. A. Owen, J. Torkington, et al., "Long Term Outcome Following Loose-Seton Technique for External Sphincter Preservation in Complex Anal Fistulae," British Journal of Surgery, Vol. 91, No. 4, 2004, pp. 476-480. doi: $10.1002 /$ bjs.4466

[5] A. Theerapol, B. Y. J. So and S. S. Ngoi, "Routine Use of Setons for the Treatment of Anal Fistulae," Singapore Medical Journal, Vol. 43, No. 6, 2002, pp. 305-307.

[6] G. N. Buchanan, C. I. Bartram, R. K. Phillips, et al., "Efficacy of Fibrin Sealant in the Management of Complex Anal Fistula: A Prospective Trial," Diseases of the Colon \& Rectum, Vol. 46, No. 9, 2003, pp. 1167-1174. doi:10.1007/s10350-004-6708-9

[7] A. Eitan, M. Koliada and A. Bickel, "The Use of the Loose Seton Technique as a Definitive Treatment for Recurrent and Persistent High Trans-Sphincteric Anal Fistulas: A Long Term Outcome," Journal of Gastrointestinal Surgery, Vol. 13, No. 6, 2009, pp. 1116-1119. doi:10.1007/s11605-009-0826-6

[8] R. A. White, T. E. Eisenstat, R. J. Rubin and E. P. Salvati, "Seton Management of Complex Anorectal Fistulas in Patients with Crohn's Disease," Diseases of the Colon \& Rectum, Vol. 33, No. 7, 1990, pp. 578-579. doi:10.1007/BF02052212

[9] R. K. Pearl, J. R. Andrews, C. P. Orsay, R. I. Weisman, M. L. Prasad, R. L. Nelson, J. R. Cintron and H. Abcarian, "Role of the Seton in the Management of Anorectal Fistulas," Diseases of the Colon \& Rectum, Vol. 36, No. 6, 1993, pp. 573-577. doi:10.1007/BF02049864

[10] W. H. Isbister and A. Sanea, "The Cutting Seton: An Experience at King Faisal Specialist Hospital," Diseases of the Colon \& Rectum, Vol. 44, No. 5, 2001, pp. 722-727. doi:10.1007/BF02234574

[11] W. F. Van Tets and J. H. C. Kuijpers, "Seton Treatment of Perianal Fistula with High Anal or Rectal Opening," British Journal of Surgery, Vol. 82, No. 7, 1995, pp. 895897. doi:10.1002/bjs.1800820711

[12] J. M. Jorge and S. D. Wexner, "Etiology and Management of Fecal Incontinence," Diseases of the Colon \& Rectum, Vol. 36, No. 1, 1993, pp. 77-97. doi:10.1007/BF02050307 